\title{
Antibacterial Drug Discovery Targeting the Lipopolysaccharide Biosynthetic Enzyme LpxC
}

\author{
Alice L. Erwin \\ Erwin Consulting, Ruston, Washington 98407 \\ Correspondence: alice.erwin@rcn.com
}

The enzyme LpxC (UDP-3-O-(R-3-hydroxymyristoyl)- $\mathrm{N}$-acetylglucosamine deacetylase) is broadly conserved across Gram-negative bacteria and is essential for synthesis of lipid $A$, the membrane anchor of the lipopolysaccharides (LPSs), which are a major component of the outer membrane in nearly all Gram-negative bacteria. LpxC has been the focus of targetdirected antibiotic discovery projects in numerous pharmaceutical and academic groups for more than 20 years. Despite intense effort, no LpxC inhibitor has been approved for therapeutic use, and only one has yet reached human studies. This article will summarize the history of LpxC as a drug target and the parallel history of research on LpxC biology. Both academic and industrial researchers have used LpxC inhibitors as tool compounds, leading to increased understanding of the differing mechanisms for regulation of LPS synthesis in Escherichia coli and Pseudomonas aeruginosa.

\section{RAETZ AND THE LIPID A BIOSYNTHETIC PATHWAY}

$\mathrm{O}$ ur current understanding of the Gramnegative outer membrane is as being physically different from a cytoplasmic membrane and constituting a permeability barrier developed during the 1960s and 1970s (Leive 1974). Research on the chemical structure of lipopolysaccharide (LPS) and its synthesis was being conducted at the same time. A general outline of LPS synthesis and assembly was complete by the mid-1970s (Osborn et al. 1972). At that time, the chemical structure of lipid A was still uncertain, and none of the enzymes involved in its synthesis had been identified. The LPS transporter MsbA and the Lpt export sys- tem were not discovered until much later (Ruiz et al. 2009).

The biochemical pathway for synthesis of lipid A was determined by Christian H.R. Raetz almost singlehandedly (Raetz 1993). Each of the enzymes was discovered by research groups he directed, working successively at the University of Wisconsin (1976-1987), Merck Research Laboratories (Rahway, NJ) (1987-1993), and Duke University (1993-2011) (Dowhan 2011; Kresge et al. 2011). During the time Raetz was at Merck, his group identified the deacetylase now known as LpxC as the first committed step in lipid A synthesis (Fig. 1) (Anderson et al. 1993). Purification of the enzyme catalyzing this activity led to the recognition that it was

Editors: Lynn L. Silver and Karen Bush

Additional Perspectives on Antibiotics and Antibiotic Resistance available at www.perspectivesinmedicine.org

Copyright (C) 2016 Cold Spring Harbor Laboratory Press; all rights reserved

Advanced Online Article. Cite this article as Cold Spring Harb Perspect Med doi: 10.1101/cshperspect.a025304 


\section{A.L. Erwin}

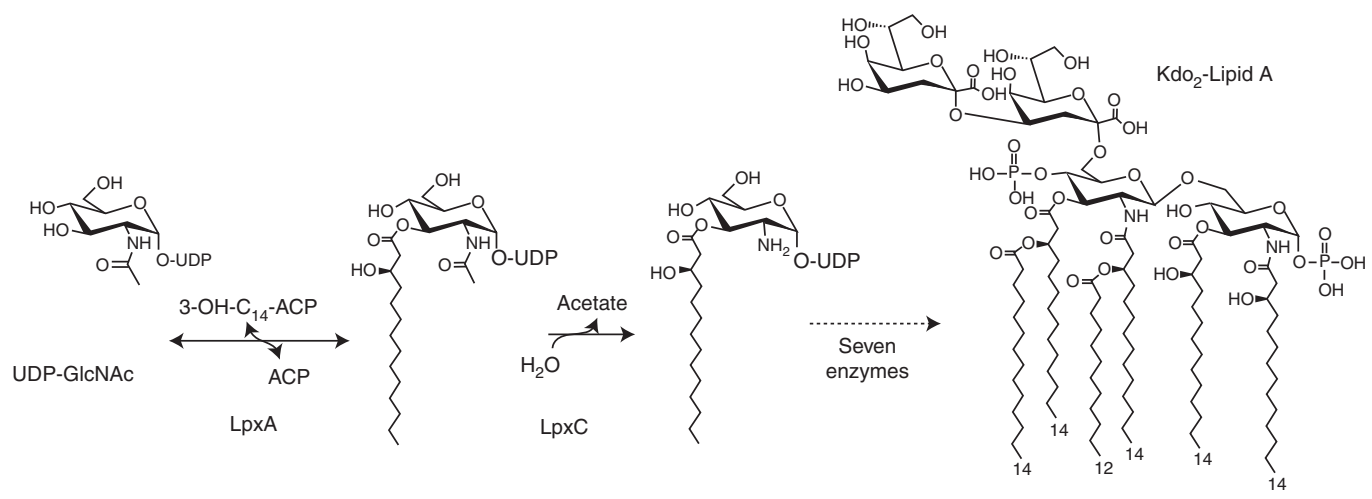

Figure 1. Lipid A biosynthesis. (Reprinted, with permission, from Mdluli et al. 2006.)

encoded by the genetic locus previously known as envA (Young et al. 1995).

\section{EARLY LpxC INHIBITORS DISCOVERED IN BACTERIAL CELL SCREENS}

The history of LpxC as a drug target began in the mid-1980s, before the discovery of the enzyme itself. The antibacterial discovery group at Merck Research Laboratories used a galE mutant of Salmonella to screen a library of chemical compounds, measuring LPS synthesis by monitoring incorporation of radiolabeled galactose into bacterial macromolecules. Among the compounds identified in the screen was the small oxazoline hydroxamic acid L-573,655 (Fig 2), which had a minimum inhibitory concentration (MIC) for wild-type Escherichia coli of $200-400 \mu \mathrm{g} / \mathrm{ml}$. This molecule was later found to be an inhibitor of $\mathrm{LpxC}$, with an $\mathrm{IC}_{50}$ for the E. coli enzyme of $8.5 \mu \mathrm{M}$. Approximately 200 analogs were synthesized, increasing potency $\sim 100$-fold. Antibacterial activity improved in parallel. The most active compound, L-161,140, had an $\mathrm{IC}_{50}$ of $0.03 \mu \mathrm{M}$ and an MIC for wildtype E. coli of $1-3 \mu \mathrm{g} / \mathrm{ml}$. None of these compounds was active against Pseudomonas aeruginosa. This was a critical issue because at the time it was believed that the market for a Gram-negative antibiotic would be very limited unless it was active against $P$. aeruginosa as well as enteric bacteria. Recognition that broad Gram-negative activity might be very difficult to achieve led to termination of this first generation of $\mathrm{LpxC}$ chemistry and subsequent publication of the work (Onishi et al. 1996; Chen et al. 1999).

British Biotech screened a library of metalloenzyme inhibitors for antibacterial activity using E. coli strain D22, an envA1 mutant. Strains carrying the point mutation envA1 (H19Y) have a defective envelope in which the outer membrane is unusually permeable to solvents and other hydrophobic compounds, conferring hypersensitivity to many antibiotics (Normark et al. 1969; Beall and Lutkenhaus 1987). Following the recognition that envA gene encodes LpxC, it had been shown that an envA1 mutant has an 18-fold reduction in LpxC activity, compared with wild-type strains (Young et al. 1995).

The partial loss of LpxC function conferred by the envA1 mutation would be expected to make the strain particularly sensitive to LpxC inhibitors, and the general hypersensitivity of the strain would increase the chances of identifying inhibitors of other targets. Two related compounds identified in this screen were found to be inhibitors of LpxC. Like the Merck series and, indeed, all potent LpxC inhibitors that have been described, both compounds are hydroxamic acid derivatives. The more active of the two, BB-78485, has an $\mathrm{IC}_{50}$ of $160 \mathrm{nM}$ versus the purified E. coli LpxC enzyme and an MIC of $1 \mu \mathrm{g} / \mathrm{ml}$ for $E$. coli. It was active against a wide variety of other Gram-negative species (MIC 2$4 \mu \mathrm{g} / \mathrm{ml}$ ), with the exception of $P$ aeruginosa $(\mathrm{MIC}>32 \mu \mathrm{g} / \mathrm{ml}$ for ATCC 27853; $4 \mu \mathrm{g} / \mathrm{ml}$ 
for a "leaky" strain, C53). As expected for inhibitors of LPS synthesis, the compounds had little or no Gram-positive activity (MIC for Staphylococcus aureus 32 or $>32 \mu \mathrm{g} / \mathrm{ml}$ ) (Clements et al. 2002).

\section{FOCUS ON INHIBITION OF $P$. aeruginosa ENZYME LED TO DISCOVERY OF BROAD- SPECTRUM LpxC INHIBITORS}

The first LpxC inhibitors able to inhibit the growth of $P$. aeruginosa were discovered by researchers from the University of Washington (UW) and Chiron, in a medicinal chemistry program funded by the Cystic Fibrosis Foundation (Andersen et al. 2011). Compounds were evaluated in an in vitro enzyme assay using LpxC from $P$. aeruginosa, rather than using the E. coli enzyme as in other early projects (Onishi et al. 1996; Raju et al. 2004). This strategy was based on the unexpected finding, discussed further below, that the reason L-161,240 does not inhibit growth of $P$. aeruginosa is that it is a poor inhibitor of the $P$. aeruginosa enzyme (Mdluli et al. 2006).

Approximately 1200 compounds were synthesized, of which the most active had MICs under $1 \mu \mathrm{g} / \mathrm{ml}$ for both $P$. aeruginosa and $E$. coli. Several compounds with MICs of $3 \mu \mathrm{g} /$ $\mathrm{ml}$ or less were found to be efficacious in mouse models of systemic infection, with $\mathrm{ED}_{50} \mathrm{~s}$ of 10 to $50 \mathrm{mg} / \mathrm{kg}$ for $P$. aeruginosa and 1.2 to $10 \mathrm{mg} / \mathrm{kg}$ for E. coli. Chiron terminated its antibacterial discovery program in early 2003, and data on the UW/Chiron LpxC compounds were presented at two conferences later that year (Anderson 2003; Erwin 2003).

\section{EXPANSION OF PHARMACEUTICAL AND ACADEMIC LpxC RESEARCH}

The reports of LpxC inhibitors active against $P$. aeruginosa led to initiation of LpxC programs at numerous companies. Most of these have not been described in the scientific literature, and public knowledge is available only through patent applications.

Between 2004 and 2013, patent applications claiming LpxC inhibitors were filed by nu- merous pharmaceutical companies, including Achaogen (South San Francisco, CA), Actelion Pharmaceuticals (Alschwil, Switzerland), AstraZeneca AB (Södertälje, Sweden), Novartis (Basel, Switzerland), Pfizer (New York), Schering Corporation (Kenilworth, NJ), Taisho Pharmaceuticals (Tokyo), and Vicuron Pharmaceuticals (New York) (Takashima et al. 2008; Benenato et al. 2010; Jain et al. 2011; Mansoor et al. 2011a; Kasar et al. 2012; Fu et al. 2014; Gauvin et al. 2015). Other companies have had varying levels of effort on LpxC programs that have not (yet) led to patent applications or to publications. The status of most of these programs is not known.

Medicinal chemistry was aided by LpxC biological research in both academic and industrial laboratories. In 2005, the Raetz laboratory and the University of Washington published the first report on the LpxC inhibitor they designated CHIR-090 (Fig. 2), identifying it as one of the most active UW/Chiron compounds (McClerren et al. 2005). This molecule, along with L-161,140 and BB-78485, was widely used as a tool compound in both academic and industrial laboratories for studies of LpxC enzymology, structural biology, and microbiology.

\section{ADVANCING TOWARD CLINICAL CANDIDATES}

From the limited biological data provided in patent applications, it does not appear that antibacterial activity per se is the major barrier to development of LpxC inhibitors as drugs. It is now routine to make compounds with in vitro antibacterial activity of MIC $1 \mu \mathrm{g} / \mathrm{ml}$ or less for both E. coli and P. aeruginosa. Data from both Achaogen and Pfizer (discussed below) show that it is possible to achieve good coverage (low $\mathrm{MIC}_{90}$ ) of both these species and of additional species associated with nosocomial infections or with cystic fibrosis. The sparse information on activity for other bacterial species suggests that there is interest in developing LpxC inhibitors that could be used for gonorrhea and for infections with biothreat agents, such as Francisella tularensis (Zhou et al. 2015b). There is some variation in antibacterial spectrum from one 
A.L. Erwin

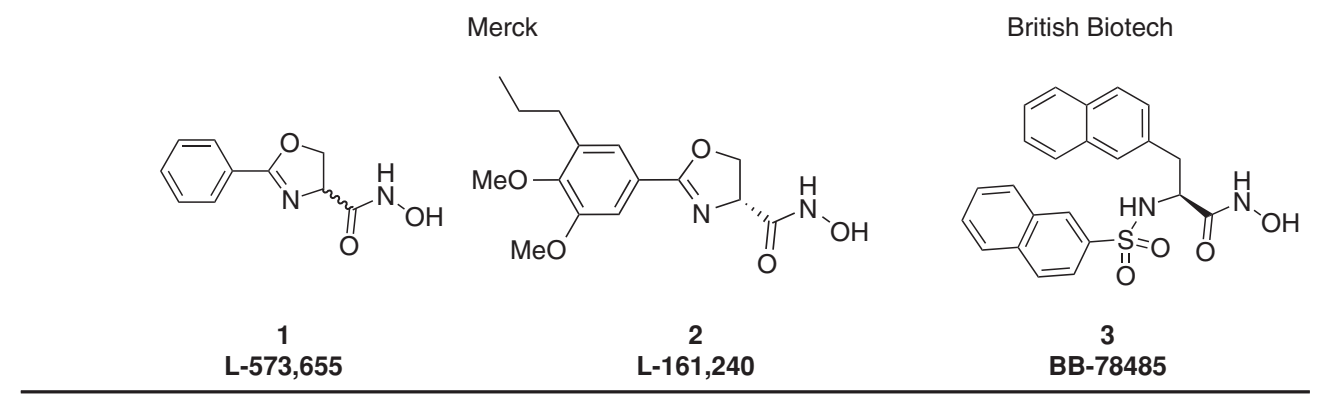

University of Washington/Chiron

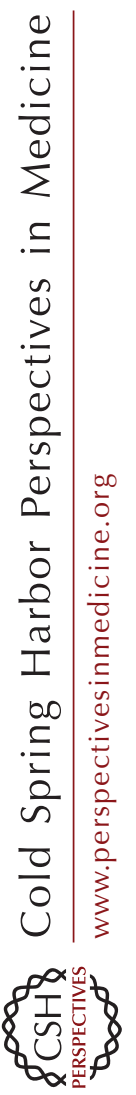<smiles>O=C(NO)[C@H]1COC(c2ccc(OC(F)(F)F)cc2)=N1</smiles>

4<smiles>CC(O)C(NC(=O)c1ccc(C#Cc2ccccc2)cc1)C(=O)O</smiles>

Ipc-004

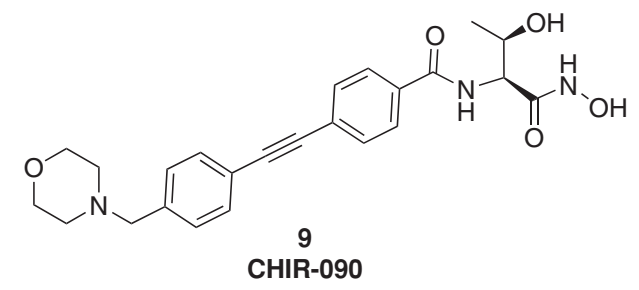

Pfizer<smiles>COc1ccc(-c2ccn(CCC(C)(C(=O)NO)S(C)(=O)=O)c(=O)c2)c(F)c1</smiles><smiles>O=C(N[C@@H](CO)C(=O)NO)c1ccc(OC(F)(F)F)cc1</smiles>

5<smiles>CC(O)C(NC(=O)c1ccc(C#CC#Cc2ccccc2)cc1)C(=O)NO</smiles>

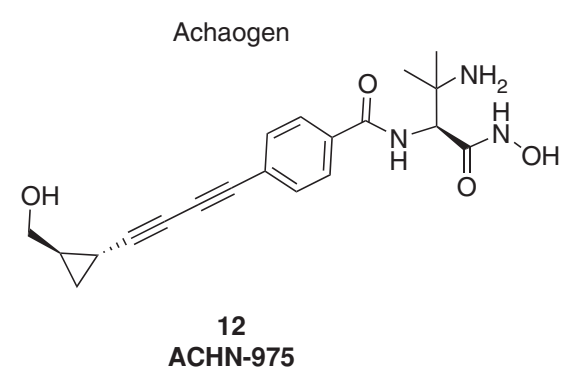

Figure 2. LpxC inhibitors. Merck: L-573,655 (compound 1) and L-161,240 (compound 2) (Onishi et al. 1996; Chen et al. 1999). British Biotech (Oxford): BB-78485 (compound 3) (Clements et al. 2002). University of Washington (UW)/Chiron (Emeryville, CA): compounds 4 and 5, previously designated 26 and 69 (Kline et al. 2002); compounds 6-10 (Andersen et al. 2011). In publications characterizing UW/Chiron compounds 7-10, they have been designated Lpc-004, CHIR-090, Lpc-009, and Lpc-011, respectively (McClerren et al. 2005; Lee et al. 2011; Liang et al. 2011). Pfizer (New York): LpxC-4 (PF-5081090) (compound 11) (Tomaras et al. 2014), previously compound 17-v (Montgomery et al. 2012), and PF1090 (Bulitta et al. 2011). Achaogen (South San Francisco): ACHN-975 (compound 12) (Kasar et al. 2012; Serio et al. 2013). 
LpxC Inhibitors

chemical series to another. For example, the isoxazole series from Pfizer includes compounds that are more active against $P$. aeruginosa than E. coli (Abramite et al. 2014).

Achieving antibacterial activity against Acinetobacter baumannii has been a challenge. A. baumannii is one of very few Gram-negative bacterial species for which LPS synthesis is not essential. Mutants in which $l p x A, l p x C$, or $l p x D$ is inactivated have been found to be viable (Moffatt et al. 2010). This initially surprising observation has led some to conclude that even very potent inhibitors of $A$. baumannii LpxC would not inhibit bacterial growth. This does not appear to be the case. Although three compounds described by Pfizer described are far less active for this species $\left(\mathrm{MIC}_{90}\right.$ $32 \mu \mathrm{g} / \mathrm{ml}$ or higher) than for E. coli and $P$. aeruginosa, this is consistent with their poor in vitro potency against the $A$. baumannii enzyme (e.g., $\mathrm{IC}_{50}$ of $183 \mathrm{nM}$ for PF-5081090) (Tomaras et al. 2014). Some of the other compounds in the same chemical series have MICs for the $A$. baumannii reference strain of $4-16 \mu \mathrm{g} / \mathrm{ml}$ (Brown et al. 2014a). More recently, patent applications from Actelion described compounds that are as active against $A$. baumannii as against $P$. aeruginosa, many with MIC $1-8 \mu \mathrm{g} / \mathrm{ml}$ or lower (Gauvin et al. 2015; Hubschwerlen et al. 2015).

Apart from antibacterial activity, there is very little information on the limitations of LpxC inhibitors with regard to other characteristics required of drug candidates, such as pharmacokinetic or toxicology profile. All of the LpxC inhibitors that have been described are hydroxamic acid derivatives, raising concerns about the toxicity that could result if mammalian metalloenzymes are also inhibited. However, several series have been shown to be tolerated in mice well enough to allow evaluation of efficacy in experimental infection. The UW/Chiron compounds have limited solubility, high protein binding, and poor pharmacokinetics. A series of publications from Pfizer described the discovery of the pyridone series through a systematic effort to improve solubility and protein binding (Brown et al. 2012; McAllister et al. 2012; Montgomery et al. 2012). This strategy led to the advanced compound PF-5081090.

\section{ACHN-975 AND PF-5081090}

The two most advanced compounds for which extensive microbiological characterization has been published are ACHN-975 from Achaogen (Kasar et al. 2012; Serio et al. 2013) and PF5081090 from Pfizer (Brown et al. 2014a; Tomaras et al. 2014). Data for these compounds are summarized in Table 1, and the chemical structures are shown in Figure 2. Both have good in vitro activity against $P$. aeruginosa and E. coli, with $\mathrm{MIC}_{90}$ of $1 \mu \mathrm{g} / \mathrm{ml}$ or less. Although both compounds are active against a variety of Gram-negative nosocomial pathogens (with the notable exception of $A$. baumannii, discussed above), they differ with respect to the cystic fibrosis pathogens. PF-5081090 has good activity against Burkholderia cepacia and Stenotrophomonas maltophilia, whereas ACHN-975 is essentially inactive against these species (Badal et al. 2013). Little is known about LpxC from either of these species, apart from a report that isolates of the $B$. cepacia complex vary widely in susceptibility to CHIR-090 (Bodewits et al. 2010). The reason for this is not known; it is possible that efflux pumps in these isolates differ in substrate specificity and that PF-5081090 is less subject to efflux by CF pathogens than is ACHN-975.

Both PF-5081090 and ACHN-975 were reported to be bactericidal and to be efficacious in mouse models of infection. For ACHN-975, cidality was described as concentration-dependent against $P$. aeruginosa and time-dependent against E. coli and Klebsiella pneumoniae (Serio et al. 2013). This was supported by the results of dose-fractionation studies in neutropenic thigh infection with each of these species (Reyes et al. 2013). PF-5081090 was reported to be efficacious in septicemia and neutropenic thigh infection models with both $P$. aeruginosa and K. pneumoniae and in a $P$. aeruginosa lung infection model in neutropenic mice (Tomaras et al. 2014).

No toxicology has been reported for PF5081090. ACHN-975 was reported to induce bradycardia in mice (Bornheim et al. 2013). Phase I evaluation in humans was initiated in 
A.L. Erwin

Table 1. Comparison of advanced compounds PF-5081090 and ACHN-975 with CHIR-090

\begin{tabular}{|c|c|c|c|c|}
\hline & & CHIR-090 & PF-5081090 & $\mathrm{ACHN}-975$ \\
\hline & & 9 & 11 & 12 \\
\hline \multicolumn{5}{|c|}{ Enzyme $\mathrm{IC}_{50}, \mathrm{nM}$} \\
\hline P. aeruginosa & & $<2.1$ & 1.1 & 0.05 \\
\hline K. pneumoniae & & N.D. & 0.069 & N.D. \\
\hline A. baumannii & & N.D. & 183 & N.D. \\
\hline \multicolumn{5}{|c|}{ Antibacterial activity, $\mathrm{MIC}$ or $\mathrm{MIC}_{90}$ (range), $\mu \mathrm{g} / \mathrm{ml}$ [number of isolates] } \\
\hline \multirow[t]{3}{*}{ P. aeruginosa } & ATCC 27853 & N.D. & N.D. & 0.2 \\
\hline & PAO1 & 1 & 0.5 & 0.06 \\
\hline & $\mathrm{MIC}_{90}$ & $4[138]$ & $1[138]$ & $0.5(0.008-0.5)[100]$ \\
\hline \multirow[t]{2}{*}{ E. coli } & ATCC 25922 & N.D. & N.D. & 0.125 \\
\hline & $\mathrm{MIC}_{90}$ & $0.25[79]$ & $0.25[79]$ & $0.5(0.03-2)[100]$ \\
\hline \multirow[t]{2}{*}{ K. pneumoniae } & ATCC 43816 & N.D. & 1 & 0.5 \\
\hline & $\mathrm{MIC}_{90}$ & N.D. & $1[98]$ & $2(0.25-4)[113]$ \\
\hline A. baumannii & $\mathrm{MIC}_{90}$ & $>64[31]$ & $>64[31]$ & $>64(4-64)[28]$ \\
\hline B. cepacia & $\mathrm{MIC}_{90}$ & $>64[30]$ & $0.5[30]$ & $16(\leq 0.03-16)[26]$ \\
\hline S. maltophilia & $\mathrm{MIC}_{90}$ & $>64[30]$ & $2[30]$ & $>16(16-16)[26]$ \\
\hline
\end{tabular}

Data for CHIR-090 and PF-5081090 (from Tomaras et al. 2014). Data for ACHN-975 compiled from 2013 ICAAC presentations (Badal et al. 2013; Serio et al. 2013); posters downloaded from www.achaogen.com/media-all on April 22, 2015.

N.D., Not determined.

2012, but clinical studies were halted because of inflammation at the injection site (see clinicaltrials.gov/ct2/show/NCT01597947; www.sec.gov/ Archives/edgar/data/1301501/00011931251402 0548/d623715ds1.htm).

As of late in 2015, ACHN-975 is the only LpxC inhibitor known to have entered clinical trials. The patents covering ACHN-975 and PF5081090 have been followed by additional patent applications from Achaogen and Pfizer, respectively (Abramite et al. 2014; Brown et al. 2014b; Linsell et al. 2014; Reilly et al. 2014; Patterson et al. 2015a,b). A clinical candidate may yet emerge from one of these companies or from one of the several other active groups of LpxC researchers. In 2013 and 2014, at least five laboratories filed new patent applications (Fu et al. 2014, 2015; Linsell et al. 2014; Chapoux et al. 2015; Cohen et al. 2015; Gauvin et al. 2015; Zhou et al. 2015a,b,c).

\section{SELECTION FOR RESISTANT MUTANTS}

Resistance to LpxC inhibitors has been studied by several groups, usually by plating bacteria onto agar containing drug at a concentration that is four times or eight times the MIC. Single-step mutations conferring resistance are rare but occur at measurable frequencies. For E. coli, separate studies of resistance to L-161,240, BB78484, or CHIR-090 each reported a frequency of $\sim 10^{-9}$ (Onishi et al. 1996; Rafanan et al. 2000; Clements et al. 2002; Zeng et al. 2013). The frequencies of resistance to PF-5081090 were reported to be $<5.0 \times 10^{-10}$ for $P$. aeruginosa and $9.6 \times 10^{-8}$ for $K$. pneumoniae (Tomaras et al. 2014). A study from Novartis obtained $P$. aeruginosa mutants resistant to CHIR-090 by serial passage on drug; frequency of resistance was not reported (Caughlan et al. 2012). Resistance mechanisms were identified for mutants in most of these studies.

For both E. coli and K. pneumoniae, the most common finding was point mutation of $f a b Z$, which encodes $R$-3-hydroxymyristoyl acyl carrier protein dehydrase (Clements et al. 2002; Zeng et al. 2013; Tomaras et al. 2014). As discussed below, it is thought that reducing the rate of phospholipid synthesis allows the cell to tolerate a reduction in LPS synthesis. For P. aeruginosa, fabZ mutants have not been observed, but several of the CHIR-090-resistant mutants 
LpxC Inhibitors

isolated by Novartis were found to have mutations in a different fatty acid biosynthetic gene, fabG (Caughlan et al. 2012).

For $P$. aeruginosa, the most commonly seen mechanism of resistance was up-regulation of multidrug efflux pumps. Novartis found $P$. aeruginosa mutants resistant to CHIR-090 with mutations in mexR or $n f x B$, genes encoding repressors for the RND pumps MexAB-OprM and MexCD-OprJ, respectively. These mutants had reduced susceptibility to several antibiotics as well as to CHIR-090, as expected for isolates overexpressing multidrug efflux pumps (Caughlan et al. 2012). Pfizer reported evidence that several of the $P$. aeruginosa isolates selected for resistance to PF-5081090 are probably also overexpressors of RND pumps. Susceptibility to PF-5081090 was restored by treatment with the efflux pump inhibitor PA $\mathrm{NN}$, and sequencing revealed mutations in efflux pump repressor genes (Tomaras et al. 2014). For enteric bacteria, efflux pump overexpression has not been reported as a mechanism of resistance to LpxC inhibitors.

Mutations that confer resistance by increasing LpxC activity have not been described in E. coli, but have been observed in P. aeruginosa. Both Pfizer and Novartis isolated resistant mutants with a C-to-A mutation 11 bp upstream of the $l p x C$ start codon and showed that laboratory constructs with this mutation were resistant. Western blots showed an increase in the amount of LpxC protein (Caughlan et al. 2012). The Pfizer report noted that the mutated base is within a recently identified small RNA designated PA4406.1 and that this is the first description of a molecular mechanism for $\mathrm{LpxC}$ regulation in $P$. aeruginosa (Tomaras et al. 2014).

Notably, very few of the resistant mutants characterized have mutations within the coding region of the $l p x C$ gene. E. coli isolates with $l p x C$ mutations were found to be resistant to early LpxC inhibitors (Rafanan et al. 2000; Clements et al. 2002), but have not been reported for the more advanced molecules. For $P$. aeruginosa, Pfizer described a M62R substitution that confers resistance to their inhibitor LpxC-2 but not to their more advanced compound PF-5081090 (Tomaras et al. 2014). Novartis reported L18V substitution in isolates of a hypermutator strain of $P$. aeruginosa selected for resistance to CHIR090 (Caughlan et al. 2012).

\section{CHEMISTRY AND STRUCTURAL BIOLOGY}

\section{Medicinal Chemistry}

Nearly all LpxC inhibitors that have been described are related to the series that was discovered by the UW/Chiron program. Early compounds in this program were designed as loose analogs of the Merck series, with a heterocyclic linker connecting an aromatic moiety to the hydroxamate warhead (Kline et al. 2002). A key observation was that acyclic precursors of oxazaline compounds were more active than their cyclic products. For example, the oxazoline shown as compound 4 in Fig. 2 had an in vitro $\mathrm{IC}_{50}$ of $5 \mu \mathrm{M}$ for $P$. aeruginosa $\mathrm{LpxC}$ and did not inhibit growth of either P. aeruginosa or E. coli. The corresponding aroylserine (compound 5) had an in vitro $\mathrm{IC}_{50}$ of $1.5 \mu \mathrm{M}$. Replacing the D-Ser ( $\alpha \mathrm{R}$ stereochemistry) with $\mathrm{L}$ Ser $(\alpha S)$ improved potency, producing the first compound active against an efflux-deficient strain of $P$. aeruginosa (MIC $12.5 \mu \mathrm{g} / \mathrm{ml}$ for mutant PAO200). Potency was increased further by addition of a methyl group to the $\beta$ carbon.

The most active threonine stereoisomer was $\alpha \mathrm{S}, \beta \mathrm{R}$ (compound 6), with an MIC of $50 \mu \mathrm{g} /$ $\mathrm{ml}$ for wild-type $P$. aeruginosa. Further exploration of L-Thr derivatives led to the molecule known as CHIR-090 as well as to dozens of other molecules with single-digit MICs for both $P$. aeruginosa and $E$. coli (Andersen et al. 2011). Antibacterial activity for E. coli emerged in this series along with $P$. aeruginosa activity, suggesting that for this scaffold, compounds had similar potency for both enzymes.

Although the UW/Chiron chemistry has not been published apart from the patent, many of the key features of the structure-activity relationship of this series are known from subsequent work published by other laboratories. Their mechanism of inhibition became apparent when the LpxC protein structure was solved. 


\section{A.L. Erwin}

The solution structure of LpxC from the thermophile Aquifex aeolicus in complex with a substrate-mimic inhibitor (TU-514) and the crystal structure of $A$. aeolicus LpxC were reported by two academic groups (Coggins et al. 2003; Whittington et al. 2003). The enzyme contains an unusual tunnel, open to solvent at both ends, into which the fatty acyl chain of the substrate is inserted. This observation suggested that the UW/Chiron inhibitors to the enzyme mimics that of the substrate, as indeed proved to be the case.

The solution structure of $A$. aeolicus LpxC in complex with CHIR-090 identified conserved amino acids that interact with the methyl and hydroxyl groups of the L-Thr, positioning the hydroxamic acid moiety in close proximity to the zinc residue in the catalytic site (Barb et al. 2007a). This is consistent with chemical data showing that L-Thr is more active than its stereoisomers (Liang et al. 2011; Hale et al. 2013).

A high-resolution crystal structure of $P$. aeruginosa LpxC with the BB-78485 inhibitor bound was first published by a group at Pfizer Global Research and Development (Mochalkin et al. 2008). With reported success in other groups following quickly, structure-based drug design is now widely used as part of LpxC medicinal chemistry programs, as described in publications from AstraZeneca, Merck, and Pfizer (Benenato et al. 2010; Mansoor et al. 2011b; Brown et al. 2012).

Nearly all reported LpxC inhibitors use hydroxamic acid as the chelating moiety. Replacing the hydroxamic acid with other warheads can reduce potency by a 100 -fold or more. However, one patent application described nonhydroxamate LpxC inhibitors with submicromolar $\mathrm{IC}_{50}$ versus the $E$. coli enzyme (Cohen et al. 2015). A press release dated December 22, 2015 from Forge Therapeutics (www.forgetherapeutics.com/news-articles) referred to a nonhydroxamate LpxC inhibitor from the same researchers as efficacious in experimental bacterial infections.

Replacement of the L-Thr with other amino acids often leads to reduction in potency (Hale et al. 2013), although not always. ACHN-975 is one example of successful replacement. Alternatives to the amino acid scaffold have been identified by several groups (Mansoor et al. 2011b; Brown et al. 2012; McAllister et al. 2012; Murphy-Benenato et al. 2014). None of the most potent compounds reaches very far into the UDP pocket of the enzyme, although several studies have attempted to gain potency through interactions in this region (Barb et al. 2009; Hale et al. 2013; Liang et al. 2013).

Many of the most active UW/Chiron compounds have two aromatic rings separated by one or two triple bonds (compounds 7-10). para or meta substitution of the distal ring by a hydrophilic group is tolerated, improving solubility without loss of activity (compounds 9 and 10). Later structural analysis showed that these molecules occupy the full length of the hydrophobic tunnel in LpxC, with the morpholino group of CHIR-090 and the amino group of Lpc-011 protruding into solvent (Barb et al. 2007a; Liang et al. 2011). Most LpxC chemistry published by other groups has used similar hydrophobic tails. The Pfizer class exemplified by PF-5081090 is unusual in being extremely potent without such an extension.

\section{Structural Basis of Species Specificity and Kinetics}

In the first description of CHIR-090, the Raetz laboratory described it as a slow, tight binder of LpxC from A. aeolicus (McClerren et al. 2005). This report increased the level of interest in LpxC, as inhibitors with slow off-rates have the possibility of being more effective in vivo. This idea was explored more recently in a study modeling $\mathrm{PK} / \mathrm{PD}$ parameters for a series of LpxC inhibitors varying in off-rate and postantibiotic effect (Walkup et al. 2015).

Similar kinetics were reported for binding of CHIR-090 to LpxC from E. coli and P. aeruginosa. In contrast, binding to LpxC from Rhizobium leguminosarum differed in being not only much weaker but rapidly reversible. Two amino acid residues within the hydrophobic tunnel were found to be critical for susceptibility and time dependence (Barb et al. 2007a,b). Later structural studies showed that the hydrophobic 
LpxC Inhibitors

tunnel of the R. leguminosarum enzyme is narrower than that of $E$. coli and the other enzymes studied. Diacetylene compounds, such as lpc009, are less bulky than CHIR-090 and are potent inhibitors of R. leguminosarum LpxC (Lee et al. 2011; Liang et al. 2011).

Most LpxC inhibitors described in the past decade are like the UW/Chiron compounds in being active versus both $E$. coli and $P$. aeruginosa. However, as noted above, this was not the case for the early inhibitors discovered by Merck and British Biotech. These compounds fail to inhibit growth of $P$. aeruginosa because they are poor inhibitors of the $P$. aeruginosa enzyme. Scientists at the PathoGenesis Corporation (later Chiron) constructed chimeric strains with controlled expression of $l_{p x C}$ genes derived from either E. coli or P. aeruginosa. Either E. coli or $P$. aeruginosa was susceptible to L-161,240 if the active $l p x C$ gene was derived from $E$. coli, but resistant if the active $l p x C$ gene was derived from P. aeruginosa (Mdluli et al. 2006).

This observation was confirmed and extended by researchers at Duke University, replacing the endogenous $l p x C$ gene in $E$. coli with that of $P$. aeruginosa and showing that the MIC of several LpxC inhibitors was dependent on the source of the gene (Table 2). They published X-ray crystal structures of the E. coli and $P$. aeruginosa enzymes in complex with various inhibitors (Lee et al. 2014). The insert I region of the E. coli enzyme contains a flexible loop $(\beta \mathrm{a}-\beta \mathrm{b})$ that flips over to accommodate bulky compounds like L-161,240 and BB-78485. The $\beta \mathrm{a}-\beta \mathrm{b}$ loop in insert I of the $P$. aeruginosa en- zyme is much more rigid. The bulky inhibitors can bind the $P$. aeruginosa enzyme, but with much lower affinity than for E. coli LpxC. Lpc009, a slender molecule similar to CHIR-090, fits readily into either type of enzyme without distorting the $\beta a-\beta b$ loop. It now appears that the issue is not that the early compounds are narrow in spectrum but that the E. coli enzyme is unusually broad. Indeed, the report describing these findings was entitled "Structural basis of the promiscuous inhibitor susceptibility of E. coli LpxC” (Lee et al. 2014).

\section{BIOLOGY OF LpxC INHIBITION}

\section{Bacterial Adaptation to Alteration of LPS Synthesis}

As indicated above, one of the most common mechanisms of resistance to $\mathrm{LpxC}$ inhibitors is mutation in $f a b Z$, which encodes $R$-3-hydroxymyristoyl acyl carrier protein dehydrase. In the first description of such mutants, Clements et al. (2002) noted that $R$-3-hydroxymyristic acid is a common precursor for both lipid A and phospholipids. They suggested that reduction of FabZ activity would divert $R$-3-hydroxymyristoyl-ACP away from phospholipid synthesis and allow the bacterial cell to maintain a normal level of LPS synthesis despite the presence of LpxC inhibitors (Clements et al. 2002).

More recent research suggests a slightly different interpretation. Zeng et al. (2013) reported that in $f a b Z$ mutants resistant to LpxC inhibitors, LpxC activity is reduced rather than

Table 2. Susceptibility of $E$. coli to LpxC inhibitors is dependent on the source of $I p x C$ gene

\begin{tabular}{lccc}
\hline & \multicolumn{3}{c}{ MIC $(\mu \mathrm{g} / \mathrm{ml})$} \\
\cline { 2 - 4 } $\begin{array}{l}\text { Species and strain } \\
\text { IpxC source }\end{array}$ & $\begin{array}{c}\text { E. coli W3110 } \\
\text { E. coli }\end{array}$ & $\begin{array}{c}\text { E. coli PA3110 } \\
\text { P. aeruginosa }\end{array}$ & $\begin{array}{c}\text { P. aeruginosa PAO1 } \\
\text { P. aeruginosa }\end{array}$ \\
\hline Inhibitor & & & \\
L-161,240 & 6.1 & $>100$ & $>100$ \\
BB-78485 & 6.1 & $>100$ & $>100$ \\
Lpc-009 & 0.05 & 0.7 & 0.7 \\
CHIR-090 & 0.2 & 1.3 & 1.6 \\
Lpc-011 & 0.03 & 0.32 & 0.32 \\
\hline
\end{tabular}

Adapted, with permission, from Lee et al. (2014).

${ }^{\mathrm{a}} \mathrm{L} p \mathrm{xC}$ replacement strain (E. coli $\operatorname{lp} x \mathrm{C}$ gene replaced by $P$. aeruginosa lp $x C$ gene). 


\section{A.L. Erwin}

increased. In contrast to envA1 mutants, in which reduced LpxC activity confers antibiotic hypersensitivity, the fabZ mutants have normal susceptibility to polymyxin. These observations suggest that in fabZ mutants, the mechanism of resistance to $\mathrm{LpxC}$ inhibition is restoration of the balance between LPS synthesis and synthesis of phospholipids, reducing flux through both pathways while maintaining the permeability barrier of the outer membrane. Overexpression of $f a b Z$ was shown to increase the amount of LpxC protein in cells, supporting the idea that FabZ and LpxC activities are coregulated. In the same study, fabZ mutants subjected to a second round of selection were found to have mutations in thrS that conferred a further fourfold increase in MIC. The double mutants grew slowly, presumably as a result of an overall reduction in protein synthesis. The investigators describe this mechanism of resistance to LpxC inhibition as rebalancing cellular homeostasis (Zeng et al. 2013).

The study reported by Zeng et al. (2013) is consistent with previous evidence that in E. coli, LpxC activity is primarily controlled at the level of protein turnover. Incubation of $E$. coli with the early LpxC inhibitor L-573,655 resulted in an increase in bacterial $\mathrm{LpxC}$ content. This response was shown to be posttranscriptional and is likely to result from a transient reduction in FtsH activity (Sorensen et al. 1996). LpxC is subject to continuous degradation by the protease FtsH. During incubation at $42{ }^{\circ} \mathrm{C}$, the mutant ftsH1 produces aberrant membrane structures within the periplasm and becomes nonviable. This phenotype can be suppressed by certain fabZ mutations (Ogura et al. 1999). Overexpression of plasmid-borne $l p x C$ is toxic, particularly if the gene is mutated to produce a protein lacking the carboxy-terminal domain recognized by FtsH (Führer et al. 2006). Overproduction of LPS is, thus, extremely detrimental to the cell.

It currently appears that in E. coli, LPS synthesis is controlled primarily at the second step in lipid A synthesis through balancing FtsH, FabZ, and LpxC activities. A recently published quantitative model of the lipid A pathway suggests that tight control at this step thwarts the effect of LpxC inhibitors. LpxK is identified as a second rate-limiting step that is not subject to regulation and might be a better drug target (Emiola et al. 2014).

In $P$. aeruginosa, much less is known about regulation of LpxC activity or of LPS synthesis in general. The $P$. aeruginosa LpxC lacks a carboxy-terminal recognition tag and is not a substrate for FtsH (Langklotz et al. 2011). As noted above, characterization of mutants resistant to LpxC inhibitors led to identification of an sRNA that appears to control LpxC activity, although the mechanism is not yet fully understood (Tomaras et al. 2014). These mutants have elevated levels of LpxC protein. Mutants with this phenotype have not been identified in enteric organisms and indeed might not be viable.

Both E. coli and P. aeruginosa are able to tolerate moderate reductions in LPS synthesis and export. The envA1 mutant discussed above is one such example. A second example is an $E$. coli mutant with a short in-frame deletion in a gene now known as $l p t D$. Because of its defect in LPS export, this mutant (imp-4213) is like envA1 in being hypersensitive to detergents and many antibiotics (Sampson et al. 1989; Ruiz et al. 2009). The P. aeruginosa mutant Z61 (ATCC 35151) has a similar phenotype (Zimmermann 1980). This strain is now known to have mutations in $l p t E$ and oprM; thus, it is defective in both outer membrane barrier and efflux (Shen et al. 2014).

\section{Effect of LpxC Inhibition on Bacterial Defenses In Vivo}

One of the potential advantages of LpxC as an antibiotic target is the possibility that sublethal concentrations of LpxC inhibitors might increase the susceptibility of bacteria to other antibiotics and perhaps also to host defenses. In in vitro "checkerboard" assays, LpxC inhibitors have been shown to be synergistic with rifampin, vancomycin, tetracycline, and other antibiotics for which the outer membrane barrier limits antibacterial activity. There have been some efforts to evaluate the extent to which this phenomenon can be exploited to improve therapy. 
LpxC Inhibitors

Co-administration of an LpxC inhibitor with another antibiotic might allow each drug to be dosed at a lower level to reduce toxicity or side effects, or allow dosing above the minimum effective level to minimize selection of resistant mutants. Pfizer reported synergy between polymyxin B nonapeptide and PF-5081090 in a mouse model of $P$. aeruginosa infection (Bulitta et al. 2011). Achaogen described synergy of both rifampin and vancomycin with LpxC inhibitors in mouse models of $P$. aeruginosa and K. pneumoniae (Patten and Armstrong 2012).

A limitation of the data obtained by in vitro selection and characterization of resistant mutants, as described above, is that they do not necessarily predict the risk for selection of resistant isolates in patients or the response of such isolates to therapy. Pfizer reported that, in mice infected with a fabZ mutant of $K$. pneumoniae and treated with PF-5081090, the AUC/MIC for stasis and 1-log kill were much lower than for mice infected with wild-type bacteria, suggesting that the fabZ mutants are more susceptible in vivo than their in vitro MICs would suggest (Tomaras et al. 2014). The fabG mutants of $P$. aeruginosa found by Novartis to be resistant to CHIR-090 had such a severe growth defect in vitro (Caughlan et al. 2012) that it seems unlikely they would survive in vivo.

To study selection of drug-resistant mutants during treatment, researchers at Achaogen infected mice with a higher inoculum than usual and treated with a suboptimal dose of an LpxC inhibitor. Tissue homogenates contained $>10^{3}$ resistant colonies per thigh. Co-administration of an additional drug (vancomycin) to improve bacterial clearance not only reduced the total number of colonies recovered but appeared to reduce the proportion of bacteria resistant to the LpxC inhibitor (Patten and Armstrong 2012). Although no characterization of the resistant bacteria was described, these observations suggest that bacterial variants with reduced susceptibility to LpxC inhibitors can emerge in vivo and that this phenomenon can be affected by dosing regimen.

The idea that sublethal concentrations of an LpxC inhibitor may sensitize bacteria to host defenses, such as complement or antimicrobial peptides, is attractive but has been little studied. Lin et al. (2012) reported that, for A. baumannii, activation of TLR4-mediated responses to endotoxin is a key aspect of virulence. They studied a Pfizer LpxC inhibitor (LpxC-1) that apparently inhibited LPS synthesis in A. baumannii (assessed by production of TLR4-activating material), although it failed to inhibit bacterial growth in vitro $(\mathrm{MIC}>512 \mu \mathrm{g} / \mathrm{ml})$. Treatment of $A$. baumannii with LpxC-1 was shown to reduce induction of cellular inflammatory responses in vitro and to increase the susceptibility of bacteria to phagocytosis. Treatment of A. baumannii-infected mice with LpxC-1 increased bacterial clearance and improved survival, with a substantial reduction in serum cytokines and tumor necrosis factor $\alpha$ (TNF- $\alpha)$ (Lin et al. 2012).

\section{CONCLUDING REMARKS}

LpxC programs have been much more successful than other target-directed antibiotic discovery efforts of the past several decades, although no compound has yet been tested in human infections. The collective efforts of academic and industrial $\mathrm{LpxC}$ researchers have added to our understanding of the regulation of LPS synthesis in E. coli and P. aeruginosa.

\section{ACKNOWLEDGMENTS}

I thank James Aggen for assistance with Figure 2. I thank the following for critical reading of the manuscript: James Aggen, Karen Bush, Cheryl Quinn, Alisa Serio, and Andrew Tomaras. I was involved in the University of Washington/Chiron LpxC program as an employee of PathoGenesis Corporation and Chiron Corporation, and am an inventor on the resulting patent (Andersen et al. 2011). I was later employed by Vertex Pharmaceuticals. I do not currently own stock in any of these companies. I have not received any financial support. The data presented here are from sources that are publicly available, although in some cases not peer-reviewed (e.g., conference presentations and patent applications). 


\section{A.L. Erwin}

\section{REFERENCES}

Abramite JA, Brown MF, Chen JM, Melnick MJ, Montgomery JI, Reilly U. 2014. Isoxazole derivatives useful as antibacterial agents. U.S. Patent 2014024690 (A1).

Andersen NH. 2003. Design of LpxC, UDP-(3-O-acyl)$\mathrm{N}$-acetylglucosamine deacetylase, inhibitors and their antibacterial activity. 43rd Interscience Conference on Antimicrobial Agents and Chemotherapy (ICAAC). Chicago, September 14-17.

Andersen NH, Bowman J, Erwin A, Harwood E, Kline T Mdluli K, Ng S, Pfister KB, Shawar R, Wagman AS, et al. 2011. Antibacterial agents. U.S. Patent 2011172174 (A1).

Anderson MS, Bull HG, Galloway SM, Kelly TM, Mohan S, Radika K, Raetz CR. 1993. UDP-N-acetylglucosamine acyltransferase of Escherichia coli. The first step of endotoxin biosynthesis is thermodynamically unfavorable. J Biol Chem 268: 19858-19865.

Badal R, Hoban D, Hackel M, Bouchillon S, Serio A. 2013. In vitro activity of ACHN-975 against 1,050 non-fermentative Gram-negative bacilli. 53rd Interscience Conference on Antimicrobial Agents and Chemotherapy (ICAAC), Abstract E-614. Denver, September 10-13.

Barb AW, Jiang L, Raetz CRH, Zhou P. 2007a. Structure of the deacetylase LpxC bound to the antibiotic CHIR-090: Time-dependent inhibition and specificity in ligand binding. Proc Natl Acad Sci 104: 18433-18438.

Barb AW, McClerren AL, Snehelatha K, Reynolds CM, Zhou P, Raetz CRH. 2007b. Inhibition of lipid A biosynthesis as the primary mechanism of CHIR-090 antibiotic activity in Escherichia coli. Biochemistry 46: 3793-3802.

Barb AW, Leavy TM, Robins LI, Guan Z, Six DA, Zhou P, Hangauer MJ, Bertozzi CR, Raetz CRH. 2009. Uridinebased inhibitors as new leads for antibiotics targeting Escherichia coli LpxC. Biochemistry 48: 3068-3077.

Beall B, Lutkenhaus J. 1987. Sequence analysis, transcriptional organization, and insertional mutagenesis of the envA gene of Escherichia coli. J Bacteriol 169: 5408-5415.

Benenato K, Choy A, Hale M, Hill P, Marone V, Miller M. 2010. Hydroxamic acid derivatives as Gram-negative antibacterial agents. Patent WO2010100475 (A1).

Bodewits K, Raetz CR, Govan JR, Campopiano DJ. 2010. Antimicrobial activity of CHIR-090, an inhibitor of lipopolysaccharide biosynthesis, against the Burkholderia cepacia complex. Antimicrob Agents Chemother 54: 35313533.

Bornheim L, McKinnell J, Fuchs-Knotts T, Boggs J, Kostrub CF. 2013. Preclinical safety evaluation of the novel LpxC inhibitor ACHN-975 in rat and monkey. 53rd Interscience Conference on Antimicrobial Agents and Chemotherapy (ICAAC), Abstract F-1231. Denver, September 10-13.

Brown MF, Reilly U, Abramite JA, Arcari JT, Oliver R, Barham RA, Che Y, Chen JM, Collantes EM, Chung SW, et al. 2012. Potent inhibitors of LpxC for the treatment of Gram-negative infections. J Med Chem 55: 914-923.

Brown MF, Che Y, Marfat A, Melnick MJ, Montgomery JI, Reilly U. 2014a. N-link hydroxamic acid derivatives useful as antibacterial agents. U.S. Patent 2014343031 (A1).

Brown MF, Chen JM, Melnick MJ, Montgomery JI, Reilly U. 2014b. Imidazole, pyrazole, and triazole derivatives useful as antibacterial agents. U.S. Patent 2014038975 (A1).
Bulitta JB, Kuhn M, Finegan S, George D, Barham R, Haddish-Berhane N, Betts A, Brown M, Hanna D, Montgomery J, et al. 2011. Optimizing synergy between a new LpxC inhibitor (PF1090) and polymyxin B nonapeptide (PBN) by mechanism-based modeling to efficiently support drug development. 51st Interscience Conference on Antimicrobial Agents and Chemotherapy (ICAAC), Abstract A2-1170. Chicago, September 17-20.

Caughlan RE, Jones AK, DeLucia AM, Woods AL, Xie L, Ma B, Barnes SW, Walker JR, Sprague ER, Yang X, et al. 2012. Mechanisms decreasing in vitro susceptibility to the LpxC inhibitor CHIR-090 in the Gram-negative pathogen Pseudomonas aeruginosa. Antimicrob Agents Chemother 56: 17-27.

Chapoux G, Gauvin J-C, Panchaud P, Specklin J-L, Surivet JP, Schmitt C. 2015. 1,2-dihydro-3H-pyrrolo[1,2-C]imidazol-3-one derivatives and their use as antibacterial agents. Patent WO2015132228 (A1)

Chen MH, Steiner MG, de Laszlo SE, Patchett AA, Anderson MS, Hyland SA, Onishi HR, Silver LL, Raetz CR. 1999. Carbohydroxamido-oxazolidines: Antibacterial agents that target lipid A biosynthesis. Bioorg Med Chem Lett 9: 313-318.

Clements JM, Coignard F, Johnson I, Chandler S, Palan S, Waller A, Wijkmans J, Hunter MG. 2002. Antibacterial activities and characterization of novel inhibitors of LpxC. Antimicrob Agents Chemother 46: 1793-1799.

Coggins BE, Li X, McClerren AL, Hindsgaul O, Raetz CRH, Zhou P. 2003. Structure of the LpxC deacetylase with a bound substrate-analog inhibitor. Nat Struct Biol 10: 645-651.

Cohen SM, Puerta DT, Perez C. 2015. Inhibitors of LpxC. Patent WO2015085238 (A1)

Dowhan W. 2011. The Raetz pathway for lipid A biosynthesis: Christian Rudolf Hubert Raetz, MD PhD, 19462011. J Lipid Res 52: 1857-1860.

Emiola A, George J, Andrews SS. 2014. A complete pathway model for lipid A biosynthesis in Escherichia coli. PLoS ONE 10: e0121216.

Erwin AL. 2003. LpxC inhibitors: New class of antibiotics for serious Gram-negative infections. In 8th International Antibacterial Drug Discovery \& Development Summit, Princeton, NJ, March 24-25.

Fu J, Karur S, Madera AM, Pecchi S, Sweeney ZK, Tjandra M, Yifru A. 2014. Hydroxamic acid derivatives as Lpxc inhibitors for the treatment of bacterial infections. Patent WO2014160649 (A1).

Fu J, Lee P, Madera AM, Sweeney ZK. 2015. Oxazolidinone hydroxamic acid compounds for the treatment of bacterial infections. Patent WO2015066413 (A1).

Führer F, Langklotz S, Narberhaus F. 2006. The C-terminal end of LpxC is required for degradation by the FtsH protease. Mol Microbiol 59: 1025-1036.

Gauvin J-C, Mirre A, Ochala E, Surivet J-P. 2015. Antibacterial 2H-indazole derivatives. Patent WO2015036964 (A1).

Hale MR, Hill P, Lahiri S, Miller MD, Ross P, Alm R, Gao N, Kutschke A, Johnstone M, Prince B, et al. 2013. Exploring the UDP pocket of LpxC through amino acid analogs. Bioorg Med Chem Lett 23: 2362-2367. 
Hubschwerlen C, Ochala E, Specklin J-L, Surivet J-P. 2015. Antibacterial $1 \mathrm{H}$-indazole and $1 \mathrm{H}$-indole derivatives. Patent WO2015091741 (A1).

Jain R, Gordeev M, Lewis J, Francavilla C. 2011. N-hydroxyamide derivatives possessing antibacterial activity. Patent EP2338878 (A2).

Kasar R, Linsell MS, Aggen JB, Lu QJ, Wang D, Church T, Moser HE, Patten PA. 2012. Hydroxamic acid derivatives and their use in the treatment of bacterial infections. Patent WO2012154204 (A1).

Kline T, Andersen NH, Harwood EA, Bowman J, Malanda A, Endsley S, Erwin AL, Doyle M, Fong S, Harris AL, et al. 2002. Potent, novel in vitro inhibitors of the Pseudomonas aeruginosa deacetylase LpxC. J Med Chem 45: 3112 3129 .

Kresge N, Simoni RD, Hill RL. 2011. The lipid A assembly pathway: The work of Christian Raetz. J Biol Chem 286: e6-e8.

Langklotz S, Schakermann M, Narberhaus F. 2011. Control of lipopolysaccharide biosynthesis by $\mathrm{FtsH}$-mediated proteolysis of LpxC is conserved in enterobacteria but not in all Gram-negative bacteria. J Bacteriol 193: 1090-1097.

Lee C-J, Liang X, Chen X, Zeng D, Joo SH, Chung HS, Barb AW, Swanson SM, Nicholas RA, Li Y, et al. 2011. Speciesspecific and inhibitor-dependent conformations of LpxC: Implications for antibiotic design. Chem Biol 18: $38-47$.

Lee C-J, Liang X, Gopalaswamy R, Najeeb J, Ark ED, Toone EJ, Zhou P. 2014. Structural basis of the promiscuous inhibitor susceptibility of Escherichia coli LpxC. ACS Chem Biol 9: 237-246.

Leive L. 1974. The barrier function of the gram-negative envelope. Ann NY Acad Sci 235: 109-129.

Liang X, Lee C-J, Chen X, Chung HS, Zeng D, Raetz CRH, Li Y, Zhou P, Toone EJ. 2011. Syntheses, structures and antibiotic activities of LpxC inhibitors based on the diacetylene scaffold. Bioorg Med Chem 19: 852-860.

Liang X, Lee C-J, Zhao J, Toone EJ, Zhou P. 2013. Synthesis, structure and antibiotic activity of aryl-substituted LpxC inhibitors. J Med Chem 56: 6954-6966.

Lin L, Tan B, Pantapalangkoor P, Ho T, Baquir B, Tomaras A, Montgomery JI, Reilly U, Barbacci EG, Hujer K, et al. 2012. Inhibition of LpxC protects mice from resistant Acinetobacter baumannii by modulating inflammation and enhancing phagocytosis. mBio 3: e00312-12.

Linsell MS, Aggen JB, Dozzo P, Hildebrandt DJ, Cohen F, Kasar RA, Kane TR, Gliedt MJ, McEnroe GA. 2014. Antibacterial agents. Patent WO2014165075 (A1).

Mansoor UF, Reddy PA, Siddiqui MA. 2011a. Urea derivatives as antibacterial agents. U.S. Patent 2011212080 (A1).

Mansoor UF, Vitharana D, Reddy PA, Daubaras DL, McNicholas P, Orth P, Black T, Siddiqui MA. 2011b. Design and synthesis of potent Gram-negative specific LpxC inhibitors. Bioorg Med Chem Lett 21: 1155-1161.

McAllister LA, Montgomery JI, Abramite JA, Reilly U, Brown MF, Chen JM, Barham RA, Che Y, Chung SW, Menard CA, et al. 2012. Heterocyclic methylsulfone hydroxamic acid LpxC inhibitors as Gram-negative antibacterial agents. Bioorg Med Chem Lett 22: 6832-6838.
McClerren AL, Endsley S, Bowman JL, Andersen NH, Guan Z, Rudolph J, Raetz CRH. 2005. A slow, tight-binding inhibitor of the zinc-dependent deacetylase LpxC of lipid A biosynthesis with antibiotic activity comparable to ciprofloxacin. Biochemistry 44: 16574-16583.

Mdluli KE, Witte PR, Kline T, Barb AW, Erwin AL, Mansfield BE, McClerren AL, Pirrung MC, Tumey LN, Warrener P, et al. 2006. Molecular validation of LpxC as an antibacterial drug target in Pseudomonas aeruginosa. Antimicrob Agents Chemother 50: 2178-2184.

Mochalkin I, Knafels JD, Lightle S. 2008. Crystal structure of LpxC from Pseudomonas aeruginosa complexed with the potent BB-78485 inhibitor. Protein Sci 17: 450-457.

Moffatt JH, Harper M, Harrison P, Hale JDF, Vinogradov E, Seemann T, Henry R, Crane B, St Michael F, Cox AD, et al. 2010. Colistin resistance in Acinetobacter baumannii is mediated by complete loss of lipopolysaccharide production. Antimicrob Agents Chemother 54: 4971-4977.

Montgomery JI, Brown MF, Reilly U, Price LM, Abramite JA, Arcari J, Barham R, Che Y, Chen JM, Chung SW, et al. 2012. Pyridone methylsulfone hydroxamate LpxC inhibitors for the treatment of serious Gram-negative infections. J Med Chem 55: 1662-1670.

Murphy-Benenato KE, Olivier N, Choy A, Ross PL, Miller MD, Thresher J, Gao N, Hale MR. 2014. Synthesis, structure, and SAR of tetrahydropyran-based LpxC inhibitors. ACS Med Chem Lett 5: 1213-1218.

Normark S, Boman HG, Matsson E. 1969. Mutant of Escherichia coli with anomalous cell division and ability to decrease episomally and chromosomally mediated resistance to ampicillin and several other antibiotics. $J$ Bacteriol 97: 1334-1342.

Ogura T, Inoue K, Tatsuta T, Suzaki T, Karata K, Young K, Su LH, Fierke CA, Jackman JE, Raetz CR, et al. 1999. Balanced biosynthesis of major membrane components through regulated degradation of the committed enzyme of lipid A biosynthesis by the AAA protease FtsH (HflB) in Escherichia coli. Mol Microbiol 31: 833-844.

Onishi HR, Pelak BA, Gerckens LS, Silver LL, Kahan FM, Chen MH, Patchett AA, Galloway SM, Hyland SA, Anderson MS, et al. 1996. Antibacterial agents that inhibit lipid A biosynthesis. Science 274: 980-982.

Osborn MJ, Gander JE, Parisi E. 1972. Mechanism of assembly of the outer membrane of Salmonella typhimurium. Site of synthesis of lipopolysaccharide. J Biol Chem 247: 3973-3986.

Patten PA, Armstrong ES. 2012. Antibacterial compositions. U.S. Patent 2012283175 (A1).

Patterson B, Lu Q, Aggen J, Dozzo P, Kasar R, Linsell M, Kane T, Gliedt M, Hildebrandt D, McEnroe G, et al. 2015a. Antibacterial agents. Patent EP2847162 (A1), abstract of corresponding document WO2013170030 (A1).

Patterson BD, Lu Q, Aggen JB, Dozzo P, Kasar RA, Linsell MS, Kane TR, Gliedt MJ, Hildebrandt DJ, McEnroe Ga, et al. 2015b. Antibacterial agents. Patent EP2847168 (A1), abstract of corresponding document WO2013170165 (A1).

Raetz CR. 1993. Bacterial endotoxins: Extraordinary lipids that activate eucaryotic signal transduction. J Bacteriol 175: $5745-5753$.

Rafanan N, Lopez S, Hackbarth C, Maniar M, Margolis P, Wang W, Yuan Z, Jain R, Jacobs J, Trias J. 2000. Resistance 
A.L. Erwin

in E.coli to LpxC inhibitor L-161,240 is due to mutations in the lpxC gene. 40th Interscience Conference on Antimicrobial Agents and Chemotherapy (ICAAC), Abstract 2026. Toronto, September 17-20.

Raju B, Odowd H, Gao H, Patel D, Trias J. 2004. N-hydroxyamide derivatives possessing antibacterial activity. WO2004007444 (A2).

Reilly U, Melnick MJ, Brown MF, Plummer MS, Montgomery JI, Che Y, Price LM. 2014. Fluoro-pyridinone derivatives useful as antibacterial agents. U.S. Patent 2014057919 (A1).

Reyes N, McKinnell J, Fuchs-Knotts T, Kostrub CF, Cirz R. 2013. ACHN-975 demonstrates potent efficacy against Gram-negative bacteria in the neutropenic mouse thigh infection model. 53rd Interscience Conference on Antimicrobial Agents and Chemotherapy (ICAAC), Abstract F1229. Denver, September 10-13.

Ruiz N, Kahne D, Silhavy TJ. 2009. Transport of lipopolysaccharide across the cell envelope: The long road of discovery. Nat Rev Microbiol 7: 677-683.

Sampson BA, Misra R, Benson SA. 1989. Identification and characterization of a new gene of Escherichia coli K-12 involved in outer membrane permeability. Genetics 122: 491-501.

Serio AW, Kubo A, Lopez S, Gomez M, Corey VC, Andrews L, Schwartz MA, Kasar R, McEnroe G, Aggen J, et al. 2013. Structure, potency and bactericidal activity of ACHN-975, a first-in-class LpxC inhibitor. 53rd Interscience Conference on Antimicrobial Agents and Chemotherapy (ICAAC), Abstract F-1226. Denver, CO, September 10-13.

Shen X, Johnson NV, Jones AK, Barnes SW, Walker JR, Ranjitkar S, Woods AL, Six DA, Dean CR. 2014. Genetic characterization of the hypersusceptible Pseudomonas aeruginosa strain Z61; identification of a defect in LptE. 54th Interscience Conference on Antimicrobial Agents and Chemotherapy (ICAAC), Abstract C-105. Washington, DC, September 5-9.

Sorensen PG, Lutkenhaus J, Young K, Eveland SS, Anderson MS, Raetz CR. 1996. Regulation of UDP-3-O-[R-3-hydroxymyristoyl]- $N$-acetylglucosamine deacetylase in Es- cherichia coli. The second enzymatic step of lipid A biosynthesis. J Biol Chem 271: 25898-25905.

Takashima H, Yoshinaga M, Ushiki Y, Tsuruta R, Urabe H, Tanikawa T, Tanabe K, Baba Y, Yokotani M, Kawaguchi Y, et al. 2008. Novel hydroxamic acid derivative. Patent WO2008105515 (A1).

Tomaras AP, McPherson CJ, Kuhn M, Carifa A, Mullins L, George D, Desbonnet C, Eidem TM, Montgomery JI, Brown MF, et al. 2014. LpxC inhibitors as new antibacterial agents and tools for studying regulation of lipid A biosynthesis in Gram-negative pathogens. mBio 5: e01551-01514.

Walkup GK, You Z, Ross PL, Allen EKH, Daryaee F, Hale MR, O'Donnell J, Ehmann DE, Schuck VJA, Buurman ET, et al. 2015. Translating slow-binding inhibition kinetics into cellular and in vivo effects. Nat Chem Biol 11: 416-423.

Whittington DA, Rusche KM, Shin H, Fierke CA, Christianson DW. 2003. Crystal structure of LpxC, a zinc-dependent deacetylase essential for endotoxin biosynthesis. Proc Natl Acad Sci 100: 8146-8150.

Young K, Silver LL, Bramhill D, Cameron P, Eveland SS, Raetz CR, Hyland SA, Anderson MS. 1995. The envA permeability/cell division gene of Escherichia coli encodes the second enzyme of lipid A biosynthesis. UDP3-O-(R-3-hydroxymyristoyl)- $N$-acetylglucosamine deacetylase. J Biol Chem 270: 30384-30391.

Zeng D, Zhao J, Chung HS, Guan Z, Raetz CRH, Zhou P. 2013. Mutants resistant to LpxC inhibitors by rebalancing cellular homeostasis. J Biol Chem 288: 5475-5486.

Zhou P, Toone E, Nicholas R. 2015a. 2-Piperidinyl substituted N,3-dihydroxybutanamides. Patent WO2015024016 (A2).

Zhou P, Toone E, Nicholas R. 2015b. Antibacterial compounds. Patent WO2015024021 (A2).

Zhou P, Toone E, Nicholas R. 2015c. Substituted hydroxamic acid compounds. Patent WO2015024010 (A2).

Zimmermann W. 1980. Penetration of $\beta$-lactam antibiotics into their target enzymes in Pseudomonas aeruginosa: Comparison of a highly sensitive mutant with its parent strain. Antimicrob Agents Chemother 18: 94-100. 


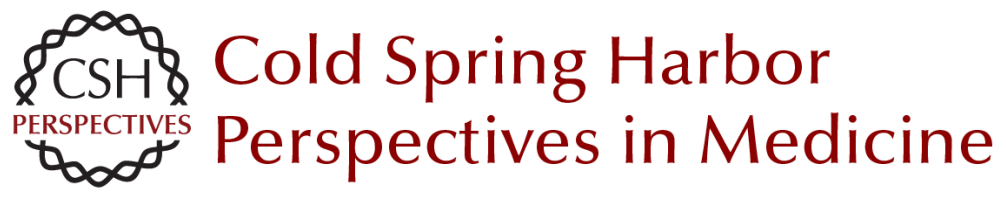

\title{
Antibacterial Drug Discovery Targeting the Lipopolysaccharide Biosynthetic Enzyme LpxC
}

\author{
Alice L. Erwin
}

Cold Spring Harb Perspect Med 2016; doi: 10.1101/cshperspect.a025304 originally published online May 27,2016

\section{Subject Collection Antibiotics and Antibiotic Resistance}

Fosfomycin: Mechanism and Resistance Lynn L. Silver

Pleuromutilins: Potent Drugs for Resistant Bugs

--Mode of Action and Resistance

Susanne Paukner and Rosemarie Riedl

Appropriate Targets for Antibacterial Drugs Lynn L. Silver

Lincosamides, Streptogramins, Phenicols, and Pleuromutilins: Mode of Action and Mechanisms of Resistance

Stefan Schwarz, Jianzhong Shen, Kristina Kadlec, et al.

Resistance to Macrolide Antibiotics in Public Health Pathogens

Corey Fyfe, Trudy H. Grossman, Kathy Kerstein, et al.

Bacterial Protein Synthesis as a Target for Antibiotic Inhibition Stefan Arenz and Daniel N. Wilson

Antibacterial Antifolates: From Development through Resistance to the Next Generation Alexavier Estrada, Dennis L. Wright and Amy C. Anderson

Antibacterial Drug Discovery Targeting the Lipopolysaccharide Biosynthetic Enzyme LpxC Alice L. Erwin
The Whys and Wherefores of Antibiotic

Resistance

Cameron R. Strachan and Julian Davies

$\beta$-Lactamases: A Focus on Current Challenges

Robert A. Bonomo

Approved Glycopeptide Antibacterial Drugs:

Mechanism of Action and Resistance

Daina Zeng, Dmitri Debabov, Theresa L. Hartsell, et al.

Mechanism of Action and Resistance to

Daptomycin in Staphylococcus aureus and

Enterococci

William R. Miller, Arnold S. Bayer and Cesar A.

Arias

Polymyxin: Alternative Mechanisms of Action and

Resistance

Michael J. Trimble, Patrik Mlynárcik, Milan Kolár, et al.

Topoisomerase Inhibitors: Fluoroquinolone

Mechanisms of Action and Resistance

David C. Hooper and George A. Jacoby

$\beta$-Lactams and $\beta$-Lactamase Inhibitors: An

Overview

Karen Bush and Patricia A. Bradford

Rifamycins, Alone and in Combination David M. Rothstein

For additional articles in this collection, see http://perspectivesinmedicine.cshlp.org/cgi/collection/ 